\title{
Formal verification of multitask hybrid systems by the OTS/CafeOBJ method
}

\author{
Masaki Nakamura $^{1} \quad$ Kazutoshi Sakakibara $^{1} \quad$ Yuki Okura $^{1} \quad$ Kazuhiro Ogata $^{2}$ \\ ${ }^{1}$ Toyama Prefectural University, $\quad 2$ Japan Advanced Institute of Science and Technology, \\ Toyama, Japan Ishikawa, Japan
}

\begin{abstract}
Hybrid systems combine both continuous and discrete behaviors. Formal descriptions of hybrid systems may help us to verify desired properties of a given system formally with computer supports. In this paper, we propose a way to describe a formal specification of a given multitask hybrid system as an observational transition system in CafeOBJ algebraic specification language and verify it by the proof score method based on equational reasoning implemented in CafeOBJ interpreter.
\end{abstract}

Keywords-component; hybrid system; algebraic specification; observational transition system; proof score method

\section{INTRODUCTION}

Formal methods are mathematically based techniques for specification and verification of software and hardware systems. Formal specification languages play important role in formal methods. CafeOBJ is an executable formal specification language, which provides specification execution based on a rewrite theory. The OTS/CafeOBJ method is a formal method in which a system is modeled as an observational transition system (OTS), its specification is described in CafeOBJ, and properties are verified formally by using specification execution function implemented in CafeOBJ, called the proof score method $[1,2]$. In our previous work [3], we have proposed a way to describe and verify multitasking real-time systems in the OTS/CafeOBJ method. A real-time system is regarded as a hybrid system where only time is its continuous data. In the literature [4], a way to describe and verify formal specifications of hybrid systems based on CafeOBJ has been proposed, however, only a single-task system is considered. In this study, we propose a way to describe a formal specification of a multitask hybrid system as an observational transition system in CafeOBJ algebraic specification language by extending the existing results, and verify it by the proof score method based on equational reasoning implemented in $\mathrm{CafeOBJ}$ interpreter.

This work was supported by JSPS KAKENHI Grant Number JP19K11842.

DOI reference number: 10.18293/SEKE2021-029

\section{PReliminaries}

\section{A Hybrid automata of a signal control system}

In this article, we consider a hybrid automaton of a simple signal control system, represented in Fig.1. The system consists of a signal and a car such that the car is prohibited from being in a specific area, between $c s_{0}$ and $c s_{1}$, while the signal is red. The specific area is called the critical section. The signal has three modes indicating its color label. Each color of the signal should be kept more than $t_{0}$ time units. The car has two modes: going and not-going. In the going mode, the car moves forward according to time advancing. The car stays there in the not-going mode. If the signal label is not green, the car cannot enter the critical section. If the signal label is changed into yellow while the car exists in the critical section, the car should keep the going mode, that is, it should not stop. Thus, if the interval $t_{0}$ is more than the time which the car needs to go through the critical section $\left(c s_{1}-c s_{0}\right)$, the car does not exist in the critical section while the signal is red.

Hybrid automata are models of hybrid systems with discrete and continuous behavior. We give a model of our simple hybrid system according to the literature [5]. Figure 2 represents a hybrid automaton

$$
\text { (Loc, Lab, Edg, X, Init, Inv, Flow, Jump) }
$$

of our simple signal control system with a single car. Locations Loc and edges Edg with labels lab are depicted as circles and arrows between them. $X$ is given as $\{$ pos, now, $l\}$. The initial values are all zero, that is, $\operatorname{Init}($ pos $)=\operatorname{Init}($ now $)=\operatorname{Init}(l)=0$, where pos and now stand for the position of the car and the current time, and $l$ is

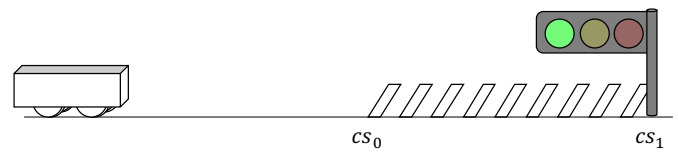

Figure 1. A signal control system 


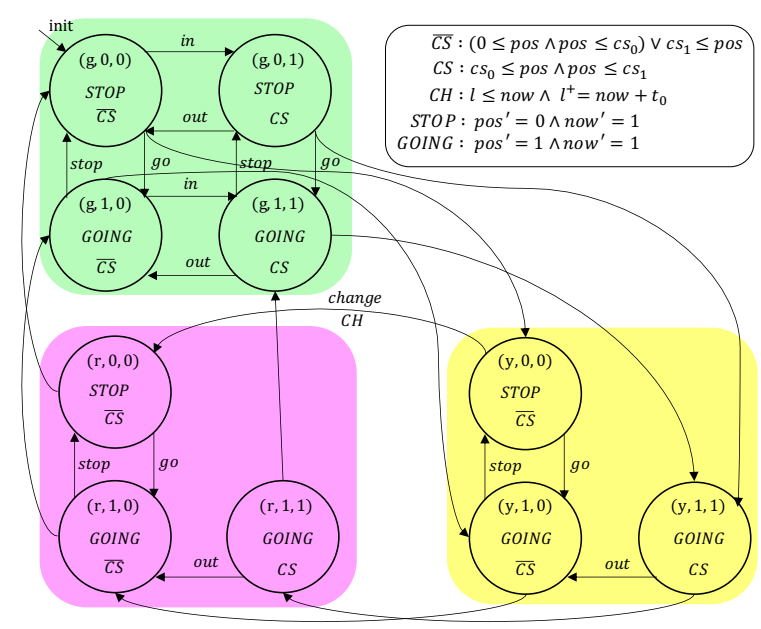

Figure 2. A hybrid automaton of a signal control system

used for the signal interval control. $L o c=\{g, y, r\} \times\{0,1\} \times$ $\{0,1\}$ is the set of locations where (label, going, cs) $\in$ Loc represents the location where the color of the signal is label, the car moves when going and the car exists in the critical section when $c s$. The top, the middle and the bottom labels in a location correspond to locations, flow conditions and invariants of the location respectively. For example, Flow $(l)=S T O P$ and $\operatorname{Inv}(l)=\overline{C S}$ for $l=(g, 0,0)$.

The flow conditions of locations are given by $S T O P$ : pos $^{\prime}=0 \wedge$ now $^{\prime}=1$ or GOING $:$ pos $^{\prime}=1 \wedge$ now $^{\prime}=1$, where $x^{\prime}$ stands for the time derivative of $x$. Since $n o w^{\prime}=1$ holds in each location, the value of now always increases according to time advancing. Thus, now keeps the elapsed time from the initial state. The value of pos is unchanged when $S T O P$ holds, and increases when GOING holds. The invariants $C S: c s_{0} \leq$ pos $\leq c s_{1}$ and $\overline{C S}:(0 \leq p o s \leq$ $\left.c s_{0}\right) \vee c s_{1} \leq$ pos mean that the car exists and does not exist in the critical section respectively.

The edges $e$ between different color's locations are labeled by change and the jump condition $\operatorname{Jump}(e)$ is given as $C H: l \leq$ now $\wedge l^{+}=$now $+t_{0}$, which means that the edge can be executed when $l \leq$ now and then the value of $l$ is updated by now $+t_{0}$. We omit some of change and $\mathrm{CH}$ in Figure 2. We also omit unchanged variables in the jump condition, that is, $x^{+}=x$ for each variable $x$.

The state transition system is obtained by a hybrid automaton, where a state $(l, v) \in S$ is a pair of a location and values of variables. From the above conditions, there is no path $s_{0} \rightarrow s_{1} \rightarrow \cdots \rightarrow s_{n}$ such that $s_{0}$ is an initial state, and $s_{n}=((r, x, y), v)$ and $c s_{0}<v(p o s)<c s_{1}$, that is, the car does not exist in the critical section when the signal is red. We call it the safety property.

Hybrid automata of plural cars can be obtained by composing the copies of the above hybrid automaton. Consider the hybrid automaton with two cars. A location is a pair $\left(l_{1}, l_{2}\right)$ where $l_{i}=\left(\right.$ label $_{i}$, going $\left._{i}, c s_{i}\right)$. There is an edge between $\left(l_{1}, l_{2}\right)$ and $\left(l_{1}^{\prime}, l_{2}^{\prime}\right)$ if there is an edge $e_{i}$ between $l_{i}$ and $l_{i}^{\prime}$ and $l_{j}=l_{j}^{\prime}(i, j \in\{1,2\}, i \neq j)$. When the number of cars increases, the state space is exponentially increases, e.g., the number of locations becomes $10^{n}$ for $n$ cars.

In the following sections, we describe OTS/CafeOBJ specifications of the above hybrid automaton with a single car and with plural cars, and give a formal proof of the safety property.

\section{$B$ The OTS/CafeOBJ method}

We introduce some notion and notation of the OTS/CafeOBJ method including the proof score method [1].

A CafeOBJ specification consists of modules. A CafeOBJ module $\mathrm{SP}=\left(\Sigma_{\mathrm{SP}}, E_{\mathrm{SP}}\right)$ consists of its signature and axioms. A signature $\Sigma_{\mathrm{SP}}=\left(S_{\mathrm{SP}}, \leq_{\mathrm{SP}}, F_{\mathrm{SP}}\right)$ consists of a set of sorts, an ordering on the sorts and an $S_{\mathrm{SP}}$-sorted set of operations. A $\Sigma_{\mathrm{SP}}$-algebra $A$ is an algebra which has a carrier set $A_{s}$ for each $s \in S_{\mathrm{SP}}$ and an operation $A_{f}: A_{s_{0}} \times \cdots \times A_{s_{n}} \rightarrow A_{s}$ for each $f \in\left(F_{\mathrm{SP}}\right)_{s_{0} \ldots s_{n} s}$. An axiom $l=r$ if $c \in E$ is a conditional equation whose both sides $l$ and $r$ of the equation are terms of a same sort and condition is a term of boolean sort constructed from the operations in $\Sigma_{\mathrm{SP}}$ and variables. A SP-algebra is a $\Sigma_{\mathrm{SP}}$-algebra which satisfies all equations in $E_{\mathrm{SP}}$. When SP has tight denotation, it denotes the initial SP-algebra. When SP has loose denotation, it denotes all SP-algebras.

The following is a loose module specifying an arbitrary set with a binary predicate.

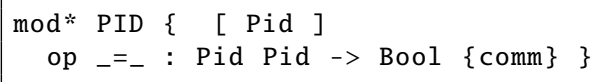

A loose module (mod*) denotes all models satisfying axioms. Module PID denotes an arbitrary set with a binary relation. The following is a tight module specifying labels of a traffic signal.

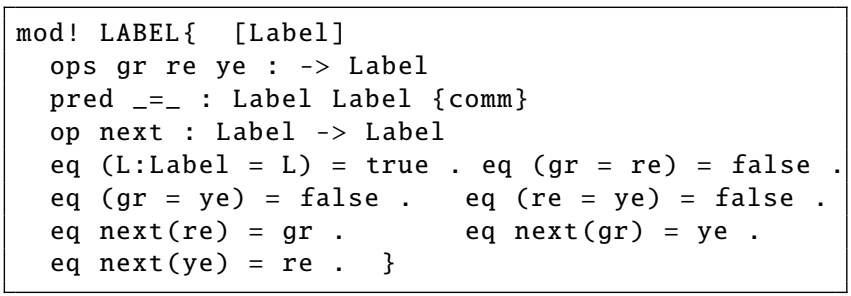

A tight module (mod!) denotes the initial model. In the initial mode, any elements of a carrier set is represented by a term constructed from its signature, and no two elements of a carrier set are equivalent unless the corresponding terms can be shown to be equal using its axioms. Module LABEL has three constant operators gr, ye and re of Label, a binary predicate $=_{-}$, and a unary operation next on Label. The first four equations define the equality predicate, which 
takes two labels and returns true if they are same, otherwise false. The unary operation next returns a next label, defined by re $\Rightarrow$ gr $\Rightarrow$ ye $\Rightarrow$ re.

An OTS/CafeOBJ specification consists of data modules and a system module. Modules LOC and PID are examples of data modules. A system module is given as a behavioral specification of CafeOBJ. A behavioral specification has a special sort, called a hidden sort, and special operations, called behavioral operations, whose arguments include the hidden sort. A behavioral operation whose returned sort is not hidden is called an observation. A behavioral operation whose returned sort is hidden is called a transition. Two elements of the hidden sort are observationally equivalent if their observed values are equivalent for each observation. An OTS/CafeOBJ specification is a restricted behavioral specification, where observational equivalence is preserved by transitions.

The following is an OTS/CafeOBJ specification of a signal control system:

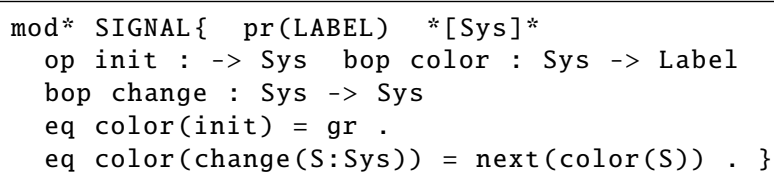

Module SIGNAL imports module LABEL with the protecting mode, where a model of the importing module includes a model of the imported module as it is. Hidden sort Sys is declared, which denotes the state space of a system to be specified. Constant init is declared as an initial state. Observation color observes a color, where term color $(s)$ represents the current color in state $s$ of the signal control system. Transition change changes a color, where term change $(s)$ represents the state obtained after changing.

The first equation color (init) = gr specifies the initial state through observation such that the initial color is green. The second equation specifies the behavior of transition change through observation. Term change(S) represents the state obtained by applying change to $\mathrm{S}$. The color of change (S) is defined as the next color of S. For example, color (change (change(init)) ) is equivalent to re.

\section{OTS/CAFEOBJ SPECIFICATIONS OF HYBRID SYSTEMS}

In this section, we introduce a way to describe OTS/CafeOBJ specifications of hybrid systems. We model signal control systems with a single car and with plural cars as hybrid automata, and describe them as OTS/CafeOBJ specifications.

\section{A An OTS/CafeOBJ specification of a signal control sys-} tem with a single car

In our OTS model of the signal control system, there are three observations for discrete locations and three observations for continuous variables. Observations color, going and $c s$ observe the value of elements of a location. Observations now, pos and $l$ observes the value of those variables. There are two kinds of transitions in OTS models: discrete and continuous transitions. Discrete transitions go, stop, in, out and change correspond to edges. A continuous transition tick $_{t}$ advances the system's time by $t \in \mathbb{Q}^{*}$.

Table 1 shows the correspondence between the hybrid automaton in Section 1 and our OTS/CafeOBJ specification.

We give a system module SIGNAL which imports the built-in module RAT of rational numbers and LABEL given in the previous section. The following is a part of module SIGNAL specifying the initial state.

$\begin{array}{ll}\text { eq now (init) }=0 . & \text { eq pos (init) }=0 . \\ \text { eq going (init) }=\text { false } . & \text { eq } \operatorname{cs}(\text { init })=\text { false } . \\ \text { eq } \operatorname{color}(\text { init })=g r . & \text { eq } l(\text { init })=\theta .\end{array}$

The initial values of now, pos and $l$ are defined as 0 . The initial values of going and $c s$ are false. The initial color is green. Module SIGNAL includes declaration ops cs 0 cs 1 tQ : -> Rat of constants $c s_{0}, c s_{1}$ and $t_{0}$.

Transition change is specified as follows:

eq $\mathrm{c}$-change $(\mathrm{S})=1(\mathrm{~S})<=\operatorname{now}(\mathrm{S})$.

ceq change $(S)=S$ if not $c$-change $(S)$.

ceq $\operatorname{color}($ change $(S))=\operatorname{next}(\operatorname{color}(S))$ if $c$-change (S).

ceq $l$ (change $(S))=$ now $(S)+$ tQ if $c$-change $(S)$. eq now (change $(S))=\operatorname{now}(S) \cdot \ldots$

The effective condition c-change (S) is defined in the first equation such that $l \leq$ now. The second (conditional) equation specifies that the state is unchanged if change is not effective. The third and fourth equations specify the updated values of color and $l$ such that color becomes the next color

TABle 1. The CORRESPONDENCE BETWEen OUR HYBRID AUtOMaton AND OTS/CAFEOBJ SPECIFICATION

\begin{tabular}{c|c} 
Hybrid Automaton & OTS/CafeOBJ specification \\
\hline \hline Loc & color, going, cs \\
\hline$E d g$ & go, stop, in, out, change \\
\hline$X$ & now, pos, 1 \\
\hline Init & init \\
\hline Inv & observed values after tick \\
\hline Flow & $\begin{array}{c}\text { effective conditions and } \\
\text { observed values after transitions }\end{array}$ \\
\hline Jump
\end{tabular}

\footnotetext{
*We assume a temporal domain is dense, that is, there is a point between any pair of points. We use rational numbers for the clock values.
} 
and $l$ is set to $t_{0}$ time later. The remaining equations specify other variables are unchanged.

Transition in is specified as follows:

eq $\mathrm{c}-$ in $(S)=(\operatorname{cs} \theta=\operatorname{pos}(S)$ and $\operatorname{color}(S)=\mathrm{gr})$

ceq $\operatorname{cs}($ in $(S))=$ true if $c-$ in $(S)$.

Transition in is effective when the car exists at $c s_{0}$ and the signal is green. When in is effective, $\operatorname{cs}(\mathrm{in}(\mathrm{S}))$ becomes true, that is, location $\left(_{-,}, 0\right)$ is changed into location $\left(_{-,}, 1\right)$. The other discrete transitions are defined similarly.

Next, we specify the continuous transition. Time advancing $t i c k_{r}$ is described as follows:

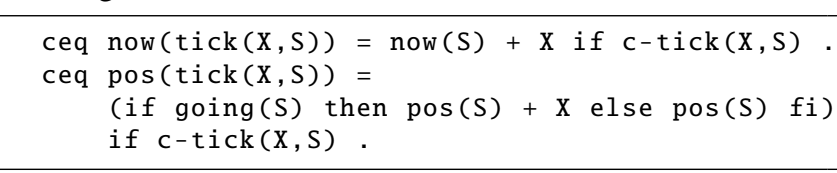

Term tick $(r, s)$ is the result state of applying tick $k_{r}$ to state $s$. Since $n o w^{\prime}=1$, the value of now increases by $1 \times r$. If we have more complex differential equations in Flow, equations become larger.

For a given $r \in \mathrm{Q}$, when tick $_{r}$ is done, the current time now increases by $r$. The position pos increases by $r$ if going is true, that is, the car moves forward. The effective condition c-tick is given by invariants of the hybrid automaton.

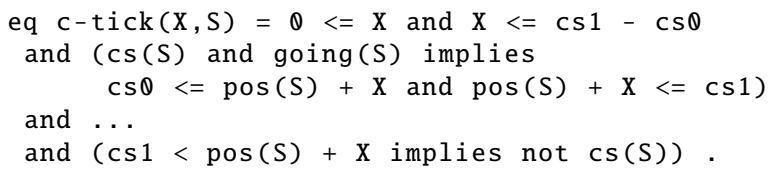

Invariants in hybrid automata should always hold, which means that time cannot advance if the invariants do not hold. Thus, the effective condition c-tick is given as the conjunction of all invariants.

\section{$B$ An OTS/CafeOBJ specification of a signal control sys- tem with plural cars}

Consider the case that more than one cars appear in our signal control system. The system is an example of multitask hybrid systems. In OTS models of multitask systems, observations and transitions related to processes are parameterized, for example, $\operatorname{pos}_{p}$ and $g o_{p}$ are an observation and a transition for a process $p$ respectively. In OTS/CafeOBJ specifications, multitask systems are described with the import of loose module PID. For example, $\operatorname{pos}_{p}$ is given by pos : Pid Sys -> Rat.

We give a system module MS which imports data modules RAT, LABEL and PID for a signal control system with plural cars. Observations and initial state of module MS are specified as follows:

$\begin{array}{ll}\text { eq now }(\text { init })=0 . & \text { eq going }(P, \text { init })=\text { false } . \\ \text { eq pos }(P, \text { init })=0 . & \text { eq } \operatorname{cs}(P, \text { init })=\text { false } . \\ \text { eq } \operatorname{color}(\text { init })=g r . & \text { eq } l(\text { init })=0 .\end{array}$

where observations pos, going, cs related to cars are parameterized. Equation pos $(\mathrm{P}$, init $)=\mathbb{0}$ means that the initial positions are zero for all cars $P$, for example. The definition of discrete transitions are modified as follows:

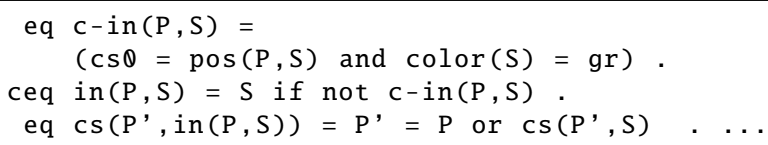

In the third equation above, term $\operatorname{cs}\left(p^{\prime}, \operatorname{in}(p, s)\right)$ means that the value of $c s_{p^{\prime}}$ at the result state of applying $i n_{p}$ to state $s$ for processes $p$ and $p^{\prime}$. Thus, when $p=p^{\prime}$, it is true otherwise it is unchanged.

The effective condition of tick $r_{r}$ should check all invariants for all processes. First, we parameterize $\mathrm{C}-\mathrm{tick}(\mathrm{P}, \mathrm{X}, \mathrm{S})$ by processes $\mathrm{P}$ as follows:

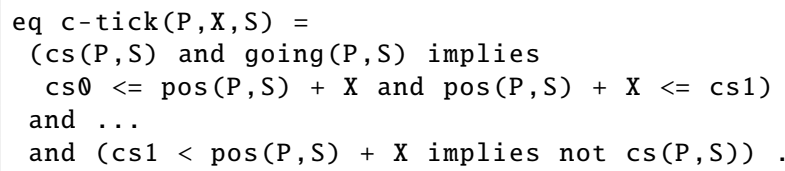

We give a way to describe multitask hybrid systems in OTS/CafeOBJ specifications which denote hybrid automata without fixed numbers of processes. We introduce a specification PSET of a set of processes:

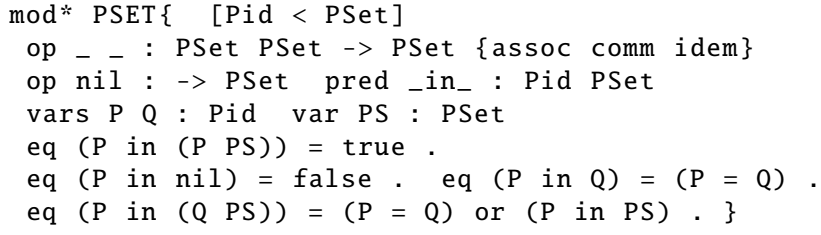

Sort PSet is declared as a super sort of Pid, and the sequence of two elements of PSet is also an element of PSet. Thus, a sequence of Pid is a term of PSet, for example, $\mathrm{p} 1$ p2 p3 is a term of PSet when p1, p2 and p3 are terms of Pid. Operation _in_denotes the membership predicate on PSet.

We also introduce an observation $p s$ which is a set of active processes. A process becomes active when it moves. After a process becomes active, it is active until it stops. The initial value of $p s$ is empty. When a car $p$ starts to move, $p s$ is updated by $p p s$. The following is a part of description related to $p s$ :

eq $p s($ init $)=$ nil. eq $p s(g \circ(P, S))=P$ ps $(S)$.

The effective condition c-tick is defined for $p s$. The effective condition of $t i c k_{r}$ is defined on $p s$ inductively:

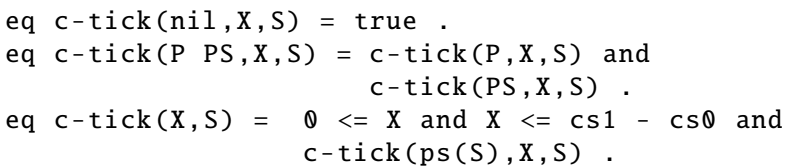

For example, c-tick (p q r $, x, s)=\mathrm{c}-\operatorname{tick}(\mathrm{p}, x, s)$ and $\mathrm{c}-\operatorname{tick}(\mathrm{q}, x, s)$ and $\mathrm{c}-\operatorname{tick}(\mathrm{r}, x, s)$. 


\section{VERIFICATION OF MULTITASK HYBRID SYSTEMS}

In this section we give a formal proof of the safety property. We declare the relationship between constants $c s_{0}, c s_{1}$ and $t_{0}$ such that $0<c s_{0}<c s_{1}$ and $c s_{1}-c s_{0} \leq t_{0}$. Then, the specification denotes all models satisfying the above condition including the test model in the previous section. First, we give a state predicate $\operatorname{inv}_{1}(p, s)$ such that the car $p$ does not exist in the critical section when the signal is red at $s$.

eq $\operatorname{inv} 1(P, S)=\operatorname{not}(\operatorname{color}(S)=$ re and

$\operatorname{cs} \theta<\operatorname{pos}(\mathrm{P}, \mathrm{S})$ and $\operatorname{pos}(\mathrm{P}, \mathrm{S})<\mathrm{cs} 1)$.

If we prove $\operatorname{inv} 1(p, s)$ for all processes $p$ and all reachable state $s$ from the initial state, the safety property holds. We denote the set of all reachable states by $R S$. We prove $\forall s \in R S . \forall p \in A_{\mathrm{Pid}} . \operatorname{inv}_{1}(p, s)$ by the induction on structure of reachable terms.

\section{A A proof passage for the induction on the reachable state space}

As the induction basis, we apply the reduction command to inv1 ( $p$, init) to prove the initial state to satisfy inv $v_{1}$, where $p$ is a fresh constant as an arbitrary element. CafeOBJ interpreter returns true, which implies the induction basis holds, that is, inv $v_{1}$ holds at the initial state.

In the induction step, we prove that each transition preserves the state predicate. We first assume an arbitrary state $\mathrm{s}$ satisfies $\operatorname{inv} 1(\mathrm{p}, \mathrm{s})$ for all processes $\mathrm{p}$. Then, under the assumption we prove inv1 (, $\mathrm{s}^{\prime}$ ) for the state s' obtained by any transition. The following is a template module for the induction step.

eq istep1(P:Pid) = inv1 $(P, s)$ implies $\operatorname{inv1}\left(P, s^{\prime}\right)$.

The proposition istep $1(\mathrm{P})$ means that the proposition inv1(P, $\left.\mathrm{S}^{\prime}\right)$ holds under the induction hypothesis $\operatorname{inv} 1(P, s)$. The following is a part of the induction steps with respect to $t i c k_{t_{1}}$, where $\mathrm{S}$ ' is obtained by applying $t i c k_{t_{1}}$ to $\mathrm{S}$.

eq $s^{\prime}=\operatorname{tick}\left(t 1, s^{\prime}\right)$ red istep $1(p)$.

If the above reduction returns true, it guarantees that implication $i n v_{1}(p, s) \Rightarrow i n v_{1}\left(t i c k_{t_{1}}(p, s)\right)$ holds. Unfortunately, it returns neither true nor false for the above proof passage. We need to give more information for proofs. A typical proof strategy is a case splitting by the effective condition as follows:

eq $\mathrm{c}-\mathrm{tick}(\mathrm{t} 1, \mathrm{~s})=$ false. eq $\mathrm{s}^{\prime}=\operatorname{tick}(\mathrm{t} 1, \mathrm{~s})$. red istep1(p).

eq $\mathrm{c}-\mathrm{tick}(\mathrm{t} 1, \mathrm{~s})=$ true . eq $\mathrm{s}^{\prime}=\operatorname{tick}(\mathrm{t} 1, \mathrm{~s})$. red istep $1(p)$.
If the above two proof passages both return true, the original proof passage is satisfied, since $\left(c-t i c k\left(t_{1}, s\right) \Rightarrow\right.$ istep $1(p)) \wedge\left(\neg c-\operatorname{tick}\left(t_{1}, s\right) \Rightarrow\right.$ istep $\left.1(p)\right) \Rightarrow$ istep $1(p)$. If it does not so, we proceed case-splitting more.

\section{B Lemma introduction}

Repeating the process of case splitting, we may face false as a returned value of the reduction command. The following is such an example.

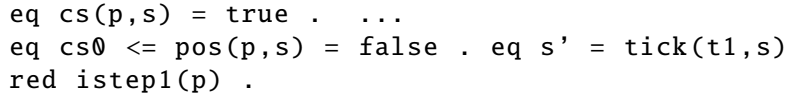

Since $c s_{p}$ is true, the position $p o s_{p}$ is greater than or equal to $c s_{0}$. The equation $\operatorname{cs} \theta<=\operatorname{pos}(\mathrm{p}, \mathrm{s})=$ false contradicts to it. Such a proof passage represents unreachable states.

For such a case, we introduce another appropriate safety property, called a lemma. We add the following lemma to the module INV and ISTEP:

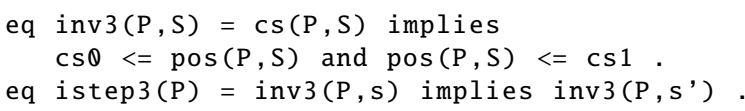

The lemma $\operatorname{inv} 3(\mathrm{P}, \mathrm{S})$ denotes that the position of car $p$ is between $c s_{0}$ and $c s_{1}$ whenever $c s_{p}$ is true. By replacing the reduction command by adding the lemma, we obtain true for the above proof passage.

red $\operatorname{inv} 3(p, s)$ implies istep $1(p)$.

Proceeding the case splitting and introducing lemma, we obtain true for all the remaining proof passages for $i n v_{1}$.

\section{Proving lemma}

We proved invariant inv $v_{1}$ for all states reachable from the initial state by the induction scheme under the assumption of some lemmata. To complete the proof, we need to show (1) the lemmata hold for the initial state, and (2) the lemmata hold for result state of applying every transition to states satisfying $i n v_{1}$ and the lemmata. In the other words, we make a proof score of the conjunction of $i n v_{1} \wedge \cdots \wedge i n v_{n}$, where the induction base is represented by $i n v_{1}(p$, init $) \wedge$ $\cdots \wedge \operatorname{inv}_{n}(p$, init) and the induction step is represented by $i n v_{1}\left(p, s^{\prime}\right) \wedge \cdots \wedge i n v_{n}\left(p, s^{\prime}\right)$ under the induction hypothesis $i n v_{1}(p, s) \wedge \cdots \wedge i n v_{n}(p, s)$. Note that in the previous section, we prove $\operatorname{inv}_{3}(p, s) \Rightarrow \operatorname{inv}_{1}(p, s) \Rightarrow \operatorname{inv} v_{1}\left(p, s^{\prime}\right)$. The formula is equivalent to $\left(i n v_{1}(p, s) \wedge i n v_{3}(p, s)\right) \Rightarrow i n v_{1}\left(p, s^{\prime}\right)$. If we prove $\left(i n v_{1}(p, s) \wedge i n v_{3}(p, s)\right) \Rightarrow i n v_{3}\left(p, s^{\prime}\right)$ for lemma $i n v_{3}$, we obtain $\left(i n v_{1}(p, s) \wedge i n v_{3}(p, s)\right) \Rightarrow \operatorname{inv} v_{1}\left(p, s^{\prime}\right) \wedge$ $i n v_{3}\left(p, s^{\prime}\right)$. 
To complete a proof of inv 1 , we make seven lemmas and 136 proof passages ${ }^{\dagger}$, all of which return true. The following is the declaration of the lemmata inv2, inv3, inv4, inv5, inv6 and inv7.

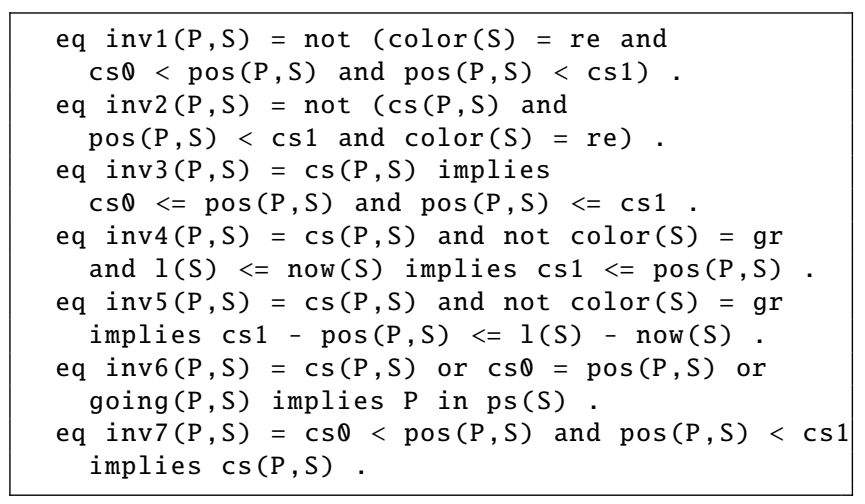

\section{Related WORK}

There are several tools for analyzing and/or verifying hybrid systems: MATLAB \& Simulink ${ }^{\ddagger}$ HSolver $^{\S}$, HyTech ${ }^{\mathbb{T}}$, KeYmaerall, PHAVer** and so on. See the literature [5] for more details. One of the most relevant tools to our study is Maude a language and tool supporting specification description and verification based on rewriting logic [6]. Both Maude and CafeOBJ are algebraic specification languages and support user-defined abstract data type specifications, which is an advantage over the other tools for hybrid systems. Real-time Maude [7] is an extension of Maude which supports formal specification and analysis of real-time and hybrid systems. HI-Maude [8] is another extension of Maude which deals with a wider range of hybrid systems, called interacting hybrid systems. System modules in Maude are based on rewriting logic, where systems transitions are described by rewrite rules. Verification in Maude is based on exhaustive searching for reachable spaces obtained by the rewrite rules. In Maude, systems with discrete and continuous variables can be described, however, only discrete time domains obtained by time sampling strategies can be verified by search and model checking.

One of our advantages against these model checking approaches is that proof scores guarantee that verified properties hold for an arbitrary number of multiple processes. To make state spaces finite, model-checking approaches should restrict the size of the system to finite. Although

\footnotetext{
${ }^{\dagger}$ Besides them, we need to add some lemmata which can be proved without induction on reachable states, e.g. eq $\operatorname{lemma} 1(P, X, S)=(P$ in $\mathrm{ps}(\mathrm{S}))$ and $\mathrm{c}-\mathrm{tick}(\mathrm{X}, \mathrm{S})$ implies $\mathrm{c}-\operatorname{tick}(\mathrm{P}, \mathrm{X}, \mathrm{S})$. We proved it by the induction on structure of terms of sort PSet.

${ }^{\ddagger}$ https://jp.mathworks.com/products/simulink.html

$\S$ http://hsolver.sourceforge.net/

${ }^{\mathbb{T}}$ https://ptolemy.berkeley.edu/projects/embedded/research/hytech/

"http://symbolaris.com/info/KeYmaera.html

${ }^{* *}$ http://www-verimag.imag.fr/\%7Efrehse/phaver_web/index.html
}

model checking is fully-automated, the proof score method is semi-automated and needs a human interaction to complete proofs. In the literature [9], automated support of making proof scores has been proposed. A case splitting phase may be automated, however, lemma discovery is heuristic and not easy to be automated.

\section{Conclusion}

We described an observational transition system of a simple signal control system with plural cars as an example of multitask hybrid systems, and verified some safety property by the proof score method. One of our future work is to apply the proposed method to practical applications of multitask hybrid systems, such as real-time operating systems, automotive control systems, intelligent transport systems, and so on.

\section{REFERENCES}

[1] K. Ogata, and K. Futatsugi, Proof scores in the OTS/CafeOBJ method, FMOODS 2003, LNCS 2884, pp.170184. Springer, 2003.

[2] K. Ogata and K. Futatsugi, Modeling and verification of real-time systems based on equations, Science of computer programming, 66(2), pp.162-180, Elsevier, 2007.

[3] M. Nakamura, S. Higashi, K. Sakakibara and K. Ogata, Formal verification of Fischer's real-time mutual exclusion protocol by the OTS/CafeOBJ method, SICE 2020, pp.1210$1215,2020$.

[4] K. Ogata, D. Yamagishi, T. Seino and K. Futatsugi, Modeling and verification of hybrid systems based on equations, DIPES 2004, pp.43-52, 2004.

[5] L. Doyen, G. Frehse, G. J. Pappas, and A. Platzer, Verification of hybrid systems, Handbook of model checking, pp.1047-1110, Springer, 2018.

[6] P. C. Ölveczky and J. Meseguer, Semantics and pragmatics of Real-Time Maude, Higher-order and symbolic computation 20, pp.161-196, Springer, 2007.

[7] D. Lepri, E. Ábrahám and P. C. Ölveczky, Timed CTL model checking in real-time Maude, WRLA 2012, LNCS 7571, pp.182-200, Springer, 2012

[8] M. Fadlisyah and P. C. Ölveczky, The HI-Maude tool, CALCO 2013, LNCS 8089, pp.322-327, Springer, 2013.

[9] D. Gaina, D. Lucanu, K. Ogata, K. Futatsugi, On automation of OTS/CafeOBJ method. SAS 2014, LNCS 8373, pp.578602, Springer, 2014. 